\title{
Tomografia computadorizada no diagnóstico da otosclerose retrofenestral
}

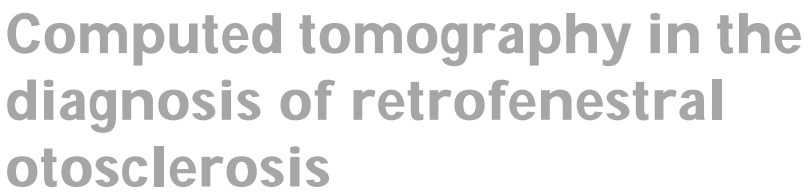

Palavras-chave: otosclerose, tomografia computadorizada por $\mathrm{Rx}$, cóclea.

Key words: otosclerosis, tomography, $\mathrm{x}$-ray computed, cochlea.

\section{Resumo / Summary}

bjetivo: Identificar os principais achados tomográficos da otosclerose retrofenestral. Método: Estudo prospectivo multicêntrico com tomografia computadorizada (TC) de ossos temporais em 54 pacientes com diagnóstico clínico e cirúrgico de otosclerose. Foi realizada uma análise tomográfica, em que foi avaliada a utilidade da TC no diagnóstico radiológico da otosclerose retrofenestral, sendo verificados os principais sítios de lesões dessa doença. Foram incluídos neste estudo 22 pacientes submetidos à TC de ossos temporais para investigação de outras afecções otológicas, que constituíram o grupo controle. Resultados: Os achados tomográficos foram bilaterais na maioria dos casos e o foco retrofenestral mais prevalente foi o foco pericoclear. Conclusões: A TC monstrou-se útil na identificação dos focos retrofenestrais. A evidência tomográfica desses focos juntamente com o envolvimento endosteal da cóclea podem sugerir uma maior atividade da doença.

\begin{abstract}
$\Lambda$
im: To identify the main tomographic findings of retrofenestral otosclerosis. Methods: A prospective and multicentric study using computed tomography (CT) of the temporal bone in 54 patients with a clinical and surgical diagnosis of otosclerosis. A tomographic analysis has been performed to evaluate the utility of CT scans in diagnosing retrofenestral otosclerosis, verifying the sites of predilection for otosclerotic lesions. In this study, 22 patients undergone CT scans of temporal bones have been included in order to investigate other otologic diseases and have constituted the control group. Results: The tomographic findings were mostly bilateral and the retrofenestral focus more prevalent was the pericochlear focus. Conclusions: The CT scan was useful in the identification of the retrofenestral focuses. The evidence of this focuses and the presence of the endosteal involvement of the cochlea can suggest a higher activity of the disease.
\end{abstract}

\footnotetext{
${ }^{1}$ Médico otorrinolaringologista pós-graduando (nível mestrado) da Disciplina de O torrinolaringologia da UNIFESP - EPM, Preceptor da residência de O torrinolaringologia do Hospital Cema.

2 Professora afiliada da Disciplina de Otorrinolaringologia da UNIFESP-EPM.

${ }^{3}$ Professor Adjunto do Departamento de Diagnóstico por Imagem da UNIFESP-EPM.

${ }^{4}$ Professor Titular da Disciplina de O torrinolaringologia da UNIFESP-EPM.

Estudo realizado nos ambulatórios de O torrinolaringologia da Universidade Federal de São Paulo - Escola Paulista de Medicina e do Hospital Cema.

Endereço para Correspondência: Andy de Oliveira Vicente - Rua Guaimbé 465 apto 10 São Paulo SP 03118-030. Tel (0xx11) 6605-3929 - E-mail: andyvicente@uol.com.br

Artigo aceito para apresentação em forma de pôster no III Congresso Triológico de Otorrinolaringologia

Artigo recebido em 06 de novembro de 2003. Artigo aceito em 11 de dezembro de 2003.
} 


\section{INTRODUÇÃO}

A cápsula ótica é composta pelas camadas endosteal, endocondral e periosteal. A otosclerose é uma doença inflamatória crônica que promove o desarranjo metabólico da camada endocondral da cápsula ótica humana caracterizandose pela reabsorção e neoformação óssea, tendo como sintoma característico a perda auditiva progressiva ${ }^{1-3}$.

A otosclerose retrofenestral caracteriza-se pela presença de focos otoscleróticos na região pericoclear, nos canais semicirculares, no meato acústico interno, no vestíbulo e nos aquedutos coclear e vestibular ${ }^{46}$.

0 estudo por imagem tem desempenhado um importante papel no diagnóstico como também no manejo clínico da otosclerose. A tomografia computadorizada de alta resolução (TCAR) é considerada o exame radiológico de eleição na avaliação das janelas labirínticas e da cápsula ótica6 ${ }^{6}$.

A TC demonstra com precisão as lesões otoscleróticas ativas ou espongióticas que são visualizadas como focos de hipodensidade ou radioluscência na cápsula ótica, podendo ser únicos, múltiplos ou confluentes, causados pelo processo de desmineralização óssea inerente a esse estágio da doença. Na doença retrofenestral, é possível evidenciar na TC uma imagem de luscência ou hipodensidade pericoclear (sinal do duplo halo), o que representa uma faixa de desmineralização endocondral da cóclea ocasionada pela confluência das lesões otoscleróticas ativas. 0 envolvimento dos canais semicirculares, meato acústico interno, vestíbulo e do endósteo da cóclea também podem ser identificados em pacientes com otosclerose retrofenestral ${ }^{6-12}$.

0 objetivo deste estudo é identificar, através da utilização da TCAR, os principais focos otoscleróticos retrofenestrais.

\section{MÉTODO}

Estudo radiológico prospectivo multicêntrico com 54 pacientes portadores de otosclerose, atendidos e selecionados nos Ambulatórios de O torrinolaringologia da Universidade Federal de São Paulo - Escola Paulista de Medicina e do Hospital Especializado CEMA. O diagnóstico de otosclerose foi obtido através da avaliação clínica (anamnese, exame otorrinolaringológico, audiometria tonal, vocal, imitanciometria) e por comprovação cirúrgica, isto é, evidência de fixação do estribo sem outra causa aparente. Todos os pacientes foram submetidos ao exame de TCAR para o estudo dos ossos temporais, totalizando 108 orelhas analisadas. Foram incluídos nesta pesquisa 22 pacientes submetidos ao exame de TC de ossos temporais para investigação de outras afecções otológicas (zumbido pulsátil, otite média crônica $(O M C)$, paralisia facial periférica, lesão no ápice petroso, Schwannoma vestibular, fratura de meato acústico externo (MAE), osteoma de MAE, malformação da cadeia ossicular), que constituíram o grupo controle.
As TCs foram realizadas em cortes axiais e coronais de 1 a $2 \mathrm{~mm}$ de espessura e $1 \mathrm{~mm}$ de incremento, com os parâmetros de 1s, 90 a 200mA, 110 a $130 \mathrm{kV}$, matriz de 512 x 512 e janela óssea entre 3500 a 4000 HU centradas em 350 a $650 \mathrm{HU}$, sem contraste endovenoso.

O s exames foram analisados e discutidos em conjunto com o radiologista. As lesões otoscleróticas foram caracterizadas como áreas de hipodensidade na cápsula ótica ou pela presença de focos de espessamento e/ ou obliteração nas janelas oval e redonda. Os focos otoscleróticos retrofenestrais foram identificados de acordo com a localização dos mesmos na cápsula ótica, como:

1 - Luscência pericoclear (LPC)

$1 \mathrm{a}-\mathrm{LPCl}$ - leve

1b - LPCm - moderada (sem o envolvimento do endósteo da cóclea)

1c - LPCS - severa (com o envolvimento de endósteo da cóclea)

2 - foco em canais semicirculares (FCSC)

$2 \mathrm{a}$ - lateral (I)

$2 b-$ superior $(s)$

$2 c$ - posterior $(p)$

3 - foco em meato acústico interno (FMAI)

4 - foco no giro basal da cóclea (FGBC)

5 - foco no giro médio da cóclea (FGMC)

6 - foco em aqueduto coclear (FAC)

7 - foco em aqueduto vestibular (FAV)

8 - foco no vestíbulo (FV)

$8 \mathrm{a}$ - parede anterior (ant)

$8 b$ - parede posterior (post)

$8 \mathrm{c}$ - parede medial $(\mathrm{m})$

Os sítios das lesões otoscleróticas citados acima foram escolhidos segundo evidências histopatológicas e radiológicas encontradas na literatura.

Durante a análise tomográfica, foi avaliada a utilidade da TC no diagnóstico radiológico da otosclerose retrofenestral. Foram avaliadas também a bilateralidade dos achados tomográficos e a eficiência dos cortes tomográficos (axial e coronal) para detectar os focos de otosclerose retrofenestral.

Para a comparação entre as distribuições dos achados tomográficos retrofenestrais nas duas orelhas, bem como para a comparação entre as distribuições desses achados obtidos nos dois diferentes cortes tomográficos, foi empregado 0 teste de homogeneidade marginal.

\section{RESULTADOS}

A população estudada foi composta por 54 pacientes (108 orelhas) com diagnóstico clínico e cirúrgico de otosclerose, sendo que $36(66,7 \%)$ eram do sexo feminino e $18(33,3 \%)$ eram do sexo masculino. A idade variou entre 15 a 59 anos, com média de 40,8 anos. 0 tempo de evolução 
da doença variou de 5 meses a 30 anos, com média de 10,7 anos. A história familiar de otosclerose foi negativa em 33 pacientes (61\%) e positiva em 21 pacientes (39\%).

Os focos otoscleróticos retrofenestrais foram evidenciados e distribuídos conforme a freqüência nos cortes axiais e coronais em orelha direita (OD) e orelha esquerda (OE) (Tabela 1).
As distribuições dos focos otoscleróticos retrofenestrais evidenciados na tomografia computadorizada estão representadas nas Tabelas 2, 3 e 4 .

A comparação entre as distribuições dos achados tomográficos nas duas orelhas e para a comparação entre as distribuições dos achados tomográficosobtidos nos dois diferentes cortes tomográficos estão representados nas Tabelas 5 e 6.

Tabela 1. Distribuição dos focos otoscleróticos em OD e OE nos cortes axial e coronal

\begin{tabular}{|c|c|c|c|c|c|c|c|c|}
\hline \multicolumn{9}{|c|}{ Cortes tomográficos em cada orelha } \\
\hline \multirow[t]{2}{*}{ Localização } & \multicolumn{2}{|c|}{ Axial OD } & \multicolumn{2}{|c|}{ Axial OE } & \multicolumn{2}{|c|}{ Coronal OD } & \multicolumn{2}{|c|}{ Coronal OE } \\
\hline & $\mathbf{N}$ & $\%$ & $\mathbf{N}$ & $\%$ & $\mathbf{N}$ & $\%$ & $\mathbf{N}$ & $\%$ \\
\hline LPC & 18 & 33,3 & 16 & 29,6 & 18 & 33,3 & 15 & 27,8 \\
\hline FGBC & 2 & 3,7 & 4 & 9,2 & 2 & 3,7 & 3 & 3 \\
\hline FAC & 2 & 3,7 & 2 & 3,7 & $\mathbf{0}$ & $\mathbf{0}$ & $\mathbf{0}$ & $\mathbf{0}$ \\
\hline FV & 2 & 3,7 & 1 & 1,85 & 0 & 0 & 0 & 0 \\
\hline FGMC & 1 & 1,85 & 1 & 1,85 & 0 & 0 & 0 & 0 \\
\hline FCNFI & 0 & 0 & 1 & 1,85 & 0 & 0 & 0 & 0 \\
\hline FCSC & 0 & 0 & 0 & 0 & 2 & 3,7 & 1 & 1,85 \\
\hline
\end{tabular}

Legendas - OD orelha direita OE orelha esquerda

LPC - luscência pericoclear;

FGBC - foco no giro basal da cóclea; FAC - foco no aqueduto coclear; FV- foco no vestíbulo;

FGMC - foco no giro médio da cóclea; FC NFI- foco no canal nervo facial

segmento labiríntico ; FC SC - foco nos canais semicirculares

Tabela 2. Distribuição da LPC na orelha direita da população estudada.

\begin{tabular}{ccccccc}
\hline & \multicolumn{5}{c}{ LPC Coronal OD } \\
& & Ausente & LPCI & LPDm & LPCs & Total \\
\hline LPC & Ausente & 36 & & & & 36 \\
Axial & LPCI & & 10 & & & 10 \\
OD & LPCm & & & 6 & & 6 \\
& LPCs & & & & 2 & 2 \\
\hline Total & & 36 & 10 & 6 & 2 & 54
\end{tabular}

Legendas : LPC = luscência pericoclear leve ; LPCI= luscência pericoclear leve

LPC $\mathbf{m}=$ luscência pericoclear moderada ; LPC s = luscência pericoclear severa
Tabela 3. Distribuição da LPC na orelha esquerda da população estudada

\begin{tabular}{ccccccc}
\hline \multicolumn{7}{c}{ LPC Coronal OE } \\
& & Ausente & LPCI & LPDm & LPCs & Total \\
\hline LPC & Ausente & 38 & \multirow{2}{*}{1} & & & 38 \\
Axial & LPCI & & 11 & 2 & & 11 \\
OD & LPCm & & & 2 & 3 & 2 \\
& LPCs & & & & 3 \\
\hline Total & & 38 & 11 & 2 & 3 & 54
\end{tabular}

Legendas : $L P C=$ luscência pericoclear leve ; $L P C I=$ luscência pericoclear leve

LPC $\mathbf{m}=$ luscência pericoclear moderada ; LPC s= luscência pericoclear severa

$O D=$ orelha direita $; O E=$ orelha esquerda

Tabela 4. Distribuição dos outros focos otoscleróticos retrofenestrais encontrados

\begin{tabular}{|c|c|c|c|c|}
\hline \multirow[t]{2}{*}{ Focos otoscleróticos } & \multicolumn{2}{|c|}{ Pacientes } & \multicolumn{2}{|c|}{ Orelhas } \\
\hline & $\mathbf{N}$ & $\%$ & $\mathbf{N}$ & $\%$ \\
\hline FMAI & 6 & 11 & 8 & 7 \\
\hline FGBC & 4 & 7,4 & 6 & 5,5 \\
\hline FAC & 2 & 3,7 & 4 & 3,7 \\
\hline FCSC & 2 & 3,7 & 3 & 2,7 \\
\hline FV & 2 & 3,7 & 3 & 2,7 \\
\hline FGMC & 1 & 1,85 & 2 & 1,85 \\
\hline FCNFI & 1 & 1,85 & 1 & 0,92 \\
\hline
\end{tabular}

Legendas: FMAI = foco no meato acústico interno ;

FGBC = foco no giro basal da cóclea;

$F A C=$ foco no aqueduto coclear $; F V=$ foco no vestíbulo ;

FGMC = foco no giro médio da cóclea

$\mathrm{FCNFI}=$ foco no canal do nervo facial porção labiríntica 
Tabela 5. Níveis descritivos da comparação entre os achados tomográficos nos cortes (a / c) da TC

\begin{tabular}{cc}
\hline Variável & Nível descritivo \\
\hline LPC OD & 1,000 \\
LPC OE & 1,000 \\
FGBC OD & 1,000 \\
FGBC OE & 0,999 \\
FMAI OE & 0,999 \\
FCSC OD & 0,999 \\
\hline
\end{tabular}

Legenda: $\mathrm{A}=$ axial; $\mathrm{C}=\mathrm{C}$ oronal

*Teste de homogeneidade marginal

Tabela 6. Avaliação da bilateralidade dos achados tomográficos

\begin{tabular}{cc} 
Variável & Nível descritivo \\
\hline LPC Axial & 0,625 \\
LPC Coronal & 0,625 \\
FGBC Axial & 0,500 \\
FGBC Coronal & 0,999 \\
FGMC Axial & 0,999 \\
FMAI Axial & 0,625 \\
FMAI Coronal & 0,999 \\
FCSC Coronal & 0,999 \\
FV Axial & 0,999 \\
FAC Axial & 1,000
\end{tabular}

*Teste de homogeneidade marginal

\section{Análise do Grupo controle}

Esse grupo era composto por 22 pacientes (31 orelhas), 12 homens (54,5\%) e 10 mulheres (45,5\%), com idade variando de 14 a 65 anos (média de 34,7 anos).

\section{Análise tomográfica do Grupo controle}

Foi observada a presença de luscência pericoclear leve (LPCl) em quatro pacientes (18,18\%), ou em seis orelhas $(19,35 \%)$. Em dois pacientes a LPCI foi bilateral. Foi visualizada área de hipotransparência na parede anterior do vestíbulo numa orelha $(3,2 \%)$ em um paciente $(4,5 \%)$.

\section{DISCUSSÃO}

Dentre os focos retrofenestrais, o que merece destaque, sem dúvida, é a luscência pericoclear, que é amplamente descrita na literatura, sendo a sua presença associada à otosclerose coclear.

Shin et al. classificaram os focos pericocleares em 2 tipos: pericocleares com envolvimento endosteal e sem envolvimento endosteal ${ }^{10}$. Para fins didáticos, graduamos, em nosso estudo, a LPC em luscência pericoclear leve (LPCI), moderada (LPCm) e severa (LPCS). A LPCI não foi considerada lesão otosclerótica em nossa casuística, apesar de não ter sido ignorada a sua ocorrência. A exclusão da LPCI como lesão otosclerótica deveu-se ao fato da mesma gerar uma interpretação radiológica subjetiva e controversa. A variabilidade anatômica interpessoal, as osteopenias, a proximidade com a medula óssea do ápice petroso (Figura $1 \mathrm{~A}$ e B), como também algumas limitações técnicas da TC são as principais causas dessa imagem tomográfica ambígua ${ }^{13,14}$. Em nosso estudo, a LPCl foi observada em 19,4\% das orelhas analisadas e foi encontrada como único achado tomográfico em um paciente (1,85\%), bilateralmente. 0 uso da TC com densitometria óssea ou até mesmo a cintilografia (SPECT) seria uma boa alternativa nesses casos duvidosos $5^{6,12,15}$ (Tabela 2 e 3). A LPCm representou a imagem de hipodensidade pericoclear (sinal do duplo halo) bem definida, porém sem promover o envolvimento da camada endosteal da cóclea (Figura 1 C e D). A LPCs foi caracterizada também como uma imagem de hipodensidade pericoclear, porém com o acometimento do endósteo da cóclea (Figura $1 \mathrm{E} \mathrm{eF}$ ). Shin etal. observaram a presença do foco pericoclear sem o envolvimento endosteal em $9,78 \%$ e o foco pericoclear com envolvimento endosteal em 3,5\% dos casos com TC positiva ${ }^{10}$. A LPCm ocorreu em $9,52 \%$ das orelhas com TC positiva e a LPCs foi evidenciada em 5,95\% destas (Tabelas 2 e 3). Se compararmos a soma das incidências da LPCm e da LPCs em nossa casuística $(15,47 \%)$ com a dos focos pericocleares observados por Shin et al. ${ }^{10}(13,28 \%)$, veremos que as mesmas são concordantes. A respeito das LPC, é importante ressaltar que esses achados tomográficos não são exclusivos da otosclerose e que outras doenças podem gerar essa hipodensidade na cápsula coclear, como a otossífilis, a osteogênese imperfeita e a doença de Paget ${ }^{6,13,16}$. Achamos que nos casos em que, na TC, são evidenciados focos difusos e altamente destrutivos na cápsula ótica, tais doenças devam ser investigadas.

O foco no meato acústico interno (FMAI) é citado na literatura radiológica e histopatológica da otosclerose de maneira sucinta, sendo descrito principalmente nos casos difusos da doença; sua incidência em estudos histológicos varia de 10 a 25\%6,17-19. Em nossa casuística, o FMAI foi encontrado em seis pacientes (11\%) ou oito orelhas $(7,4 \%)$, sendo que todos eles apresentaram múltiplos focos otoscleróticos na TC (Tabela 4 e Figura 2).

A visualização do foco no giro basal da cóclea (FGBC) na TC é, sem dúvida, relevante, uma vez que ele pode ser responsável pelo comprometimento neurossensorial da audição, principalmente nas freqüências agudas. As lesões otoscleróticas nessa região podem provocar um desarranjo estrutural acentuado, alterando a integridade endosteal da cóclea e, nos casos mais severos, é possível observar a presença de neoformação óssea intensa, inclusive com protrusão intracoclear ${ }^{6,17,20-23}$. Em nossa casuística, observamos o FGBC em 7,4\% dos pacientes (6,5\% das orelhas) (Tabela 4). Em um deles, o FG BC foi o único achado tomográfico evidente na orelha contralateral à operada (Figura $3 \mathrm{~A}$ ). 0 utro paciente apresentou um foco de hipodensidade intracoclear 


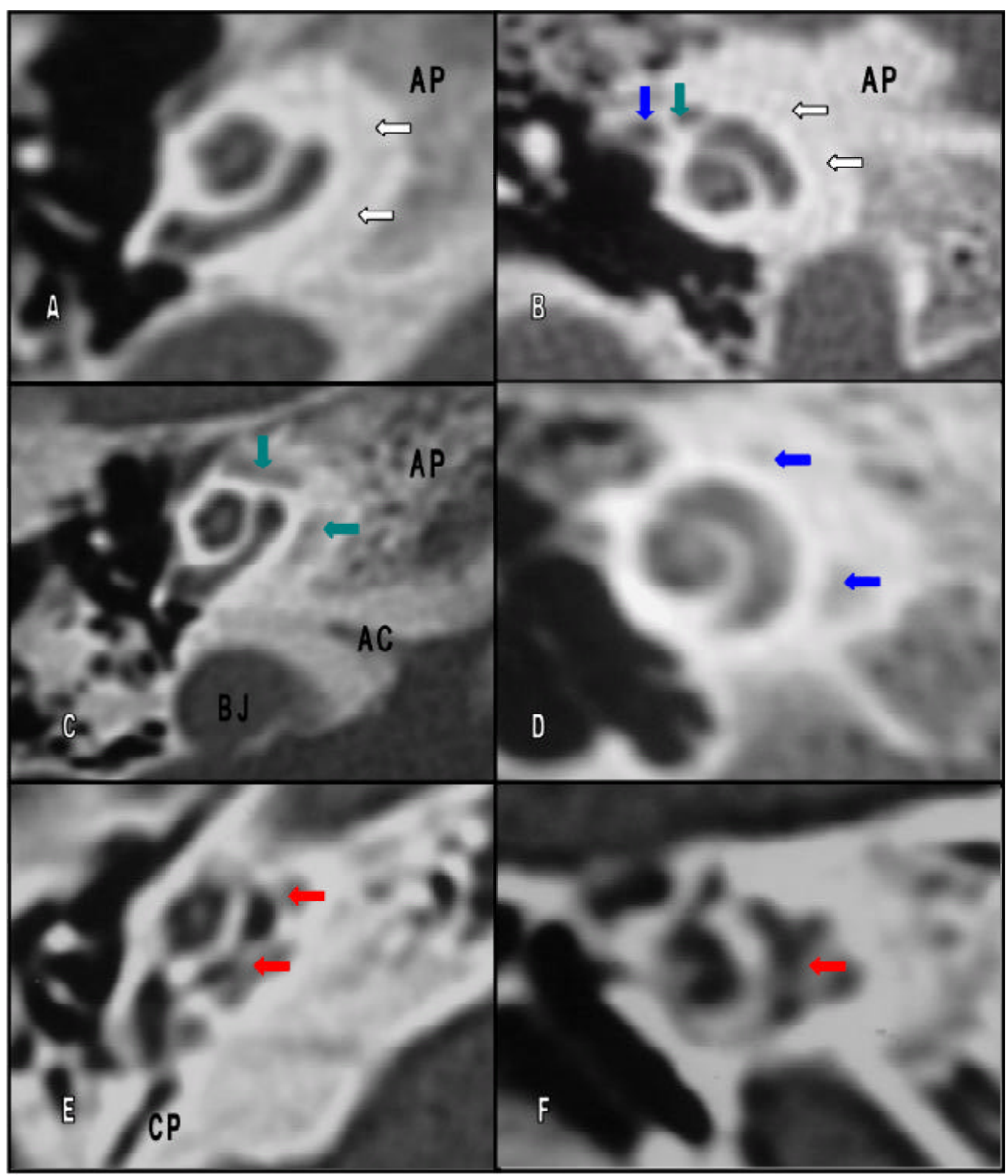

Figura 1(A-F). Tomografias computadorizadas de orelhas com luscência pericoclear (LPC). A: TC em corte axial ao nível da janela redonda demonstrando LPC leve (setas brancas); B: TC em corte coronal evidenciando LPCI (setas brancas); C: TC em corte axial demonstrando LPC moderada (setas verdes); D: TC em corte coronal mostrando a LPCm (setas azuis); E: TC em corte axial evidenciando LPC severa (setas vermelhas); F: TC em corte coronal demonstrando LPCs (seta vermelha).

Seta azul em B: segmento timpânico do nervo facial

Seta verde em B: segmento labiríntico do nervo facial

AP: ápice petroso; CP: canal semicircular posterior; BJ : bulbo da veia jugular;

$A C$ : aqueduto coclear 


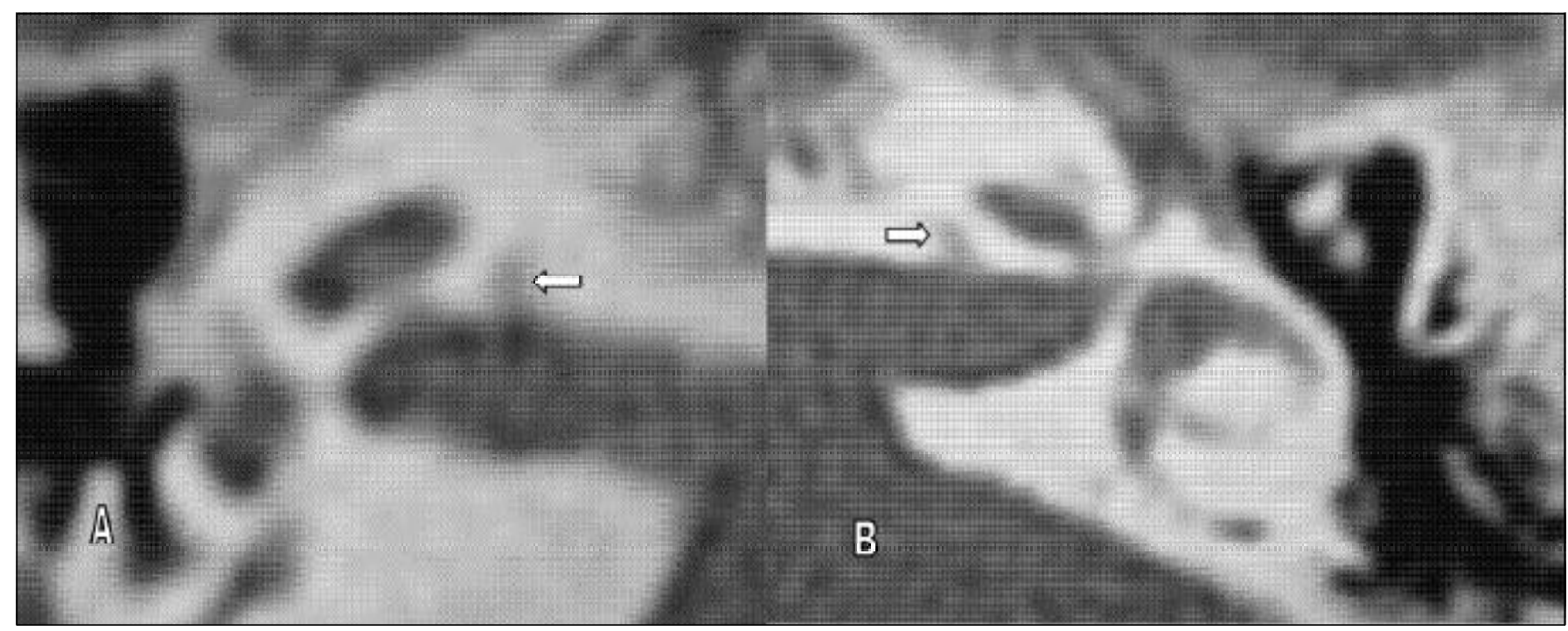

Figura 2(A-B). Tomografias computadorizadas de ossos temporais em cortes axiais, evidenciando foco de hipodensidade na parede anterior do meato acústico interno (setas)

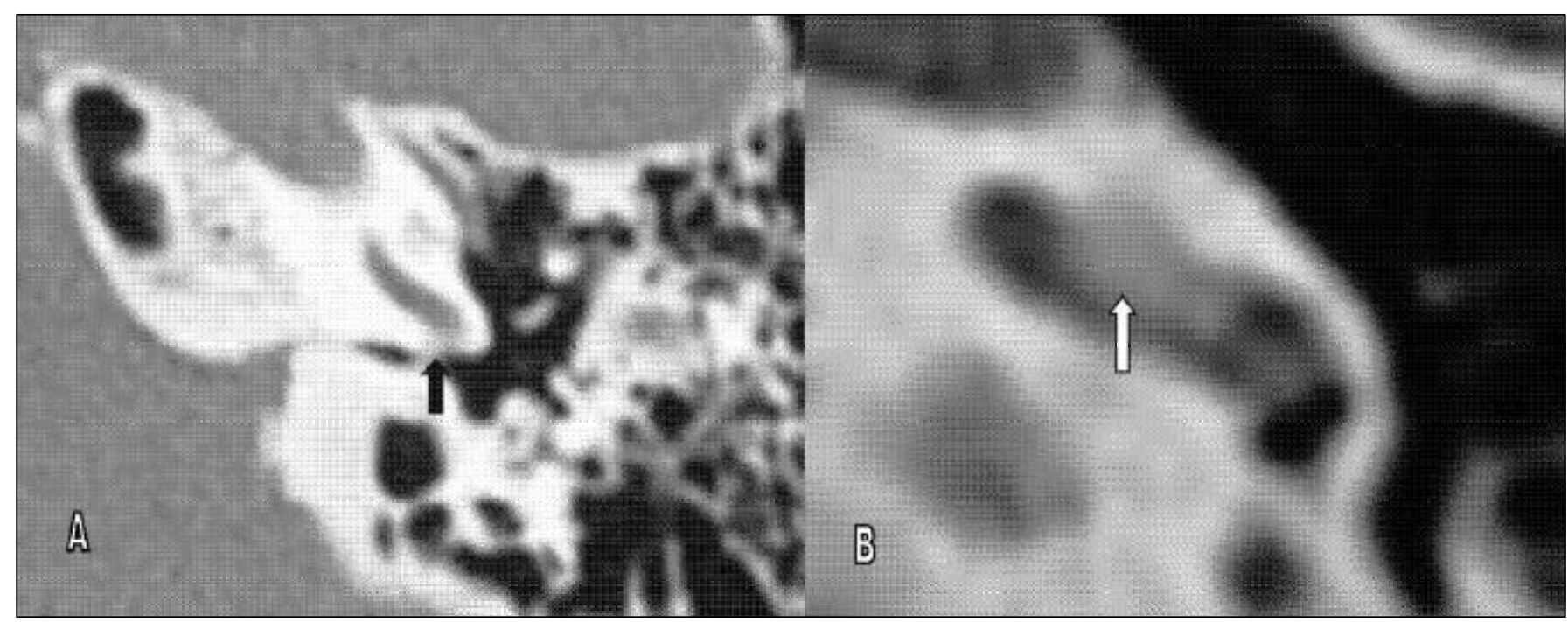

Figura 3(A-B). Tomografia computadorizada de ossos temporais em cortes axiais. A: TC evidenciando foco de hipodensidade no giro basal da cóclea (seta preta); B: TC evidenciando foco de hipodensidade na luz do giro basal da cóclea (seta branca).

na rampa timpânica em uma orelha (Figura 3B). Achamos que nos pacientes otoscleróticos com queda acentuada da via óssea nas freqüências agudas, a região do giro basal da cóclea deve ser analisada na TC com atenção.

Em nossa casuística, o foco na região próxima ao aqueduto coclear (FAC) foi demonstrado em dois pacientes $(3,7 \%)$, bilateralmente, ambos com múltiplas lesões na cápsula labiríntica, incluindo lesões na região da JR (Tabela 4 e Figura 4). Schuknecht e Barber relatam a incidência de $3 \%$ de FAC nos ossos temporais analisados e comentaram que essa lesão não poderia ser responsabilizada pelo déficit funcional da orel ha interna ${ }^{19}$. Os autores observaram ainda que as lesões obliterativas na JR poderiam bloquear o aqueduto coclear. $\mathrm{Na}$ literatura radiológica, tal achado não é discutido. Acreditamos que o envolvimento do aqueduto coclear em nossos pacientes foi secundário ao foco naJR.

Os canais semicirculares também podem ser sítios de lesões otoscleróticas. Shambaugh evidenciou focos otoscleróticos no canal semicircular lateral em 3\% das orelhas submetidas à cirurgia de fenestração ${ }^{1}$. Schuknecht observou 


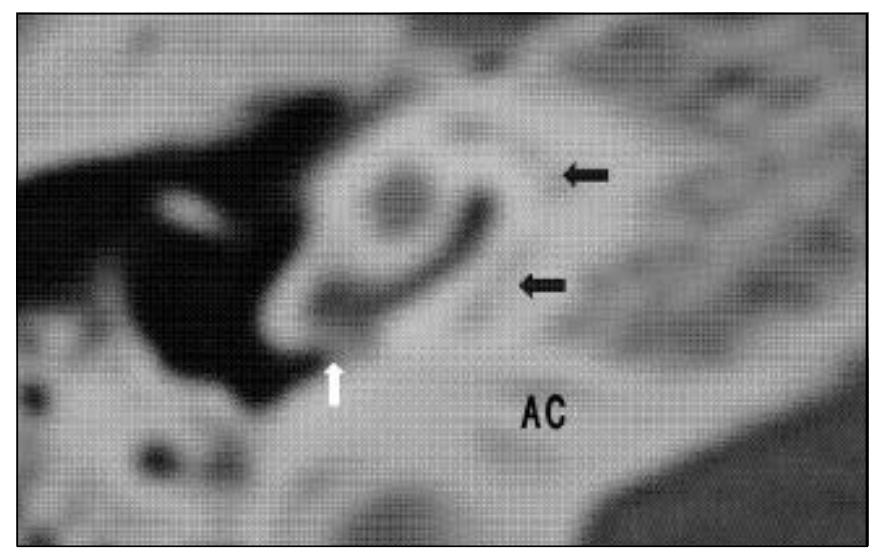

Figura 4. Tomografia computadorizada de osso temporal direito evidenciando luscência pericoclear moderada (setas preto) e foco de hipodensidade na extremidade lateral do aqueduto coclear, próximo à janela redonda (seta branca)

$A C$ : aqueduto coclear

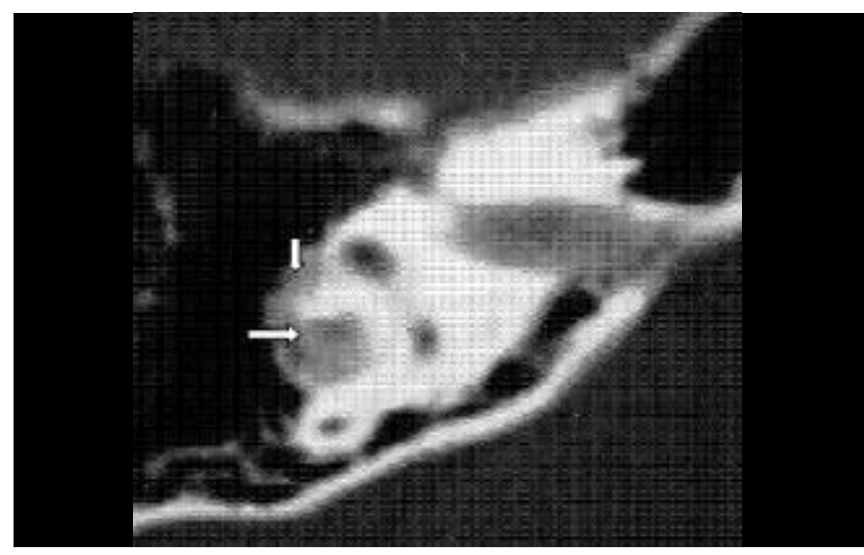

Figura 5. Tomografia de osso temporal direito em corte axial evidenciando foco de hipodensidade em canal semircular lateral (setas)

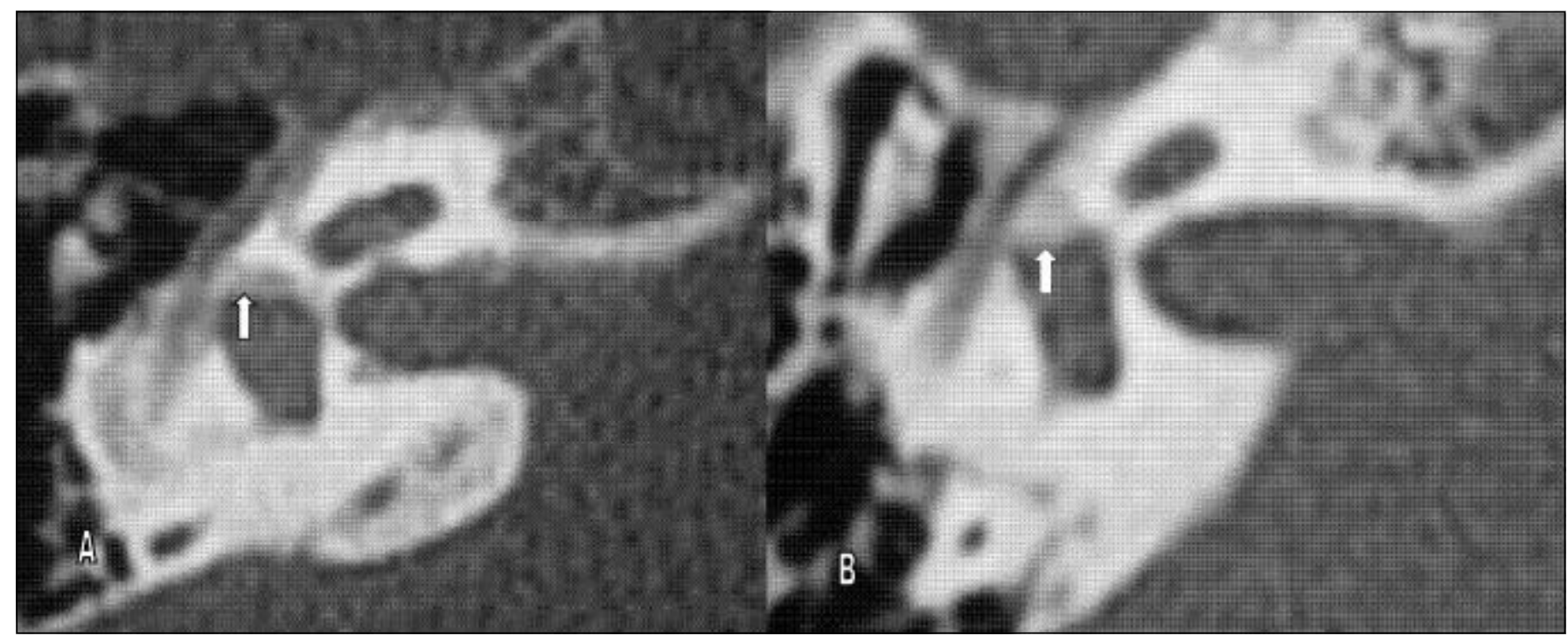

Figura 6(A e B ). Tomografias computadorizadas de ossos temporais em cortes axiais demonstrando hipodensidade na parede anterior do vestíbulo (setas)

uma incidência de $2,4 \%$ dessas lesões nos ossos temporais estudados ${ }^{24}$. Veillon et al. incluíram na sua classificação tomográfica os focos nos canais semicirculares, porém a incidência desse achado não foi citada especificamente ${ }^{25}$. Em nosso estudo, encontramos focos nos canais semicirculares (FCSC) laterais em $3,7 \%$ dos pacientes ( $2,7 \%$ das orelhas), todos com doença abrangente (Tabela 4 e Figura 5).

0 envolvimento da porção superior do vestíbulo e também o acometimento do nervo vestibular superior pelo processo otosclerótico foram identificados em ossos temporais de pacientes com otosclerose e vertigem ${ }^{26,27}$. Evidências tomográficas desses focos foram citadas em trabalhos na literatura, porém, de maneira inespecífica ${ }^{6,25}$. Em nossa casuística, evidenciamos o foco na parede anterior do vestíbulo em 3,7\% dos pacientes ( $2,7 \%$ das orelhas) (Tabela 4 e Figura 6 ). Talvez seja recomendável que, nos pacientes otoscleróticos com quadros vertiginosos, essa região seja investigada detalhadamente na TC, principalmente na sua porção ântero-superior. 
0 ápice da cóclea pode ser comprometido por lesões otoscleróticas e, segundo Schuknecht e Barber, esse é o terceiro local mais envolvido pela doença $(12,2 \%)^{19}$. Em nosso estudo, denominamos tal achado de foco no giro médio da cóclea (FGMC), o qual foi observado em 1,85\% dos nossos pacientes (Tabela 4 e Figura 7). Swartz et al. observaram na TC o comprometimento dessa região em $12 \%$ dos pacientes com otosclerose fenestral e coclear ${ }^{28}$.

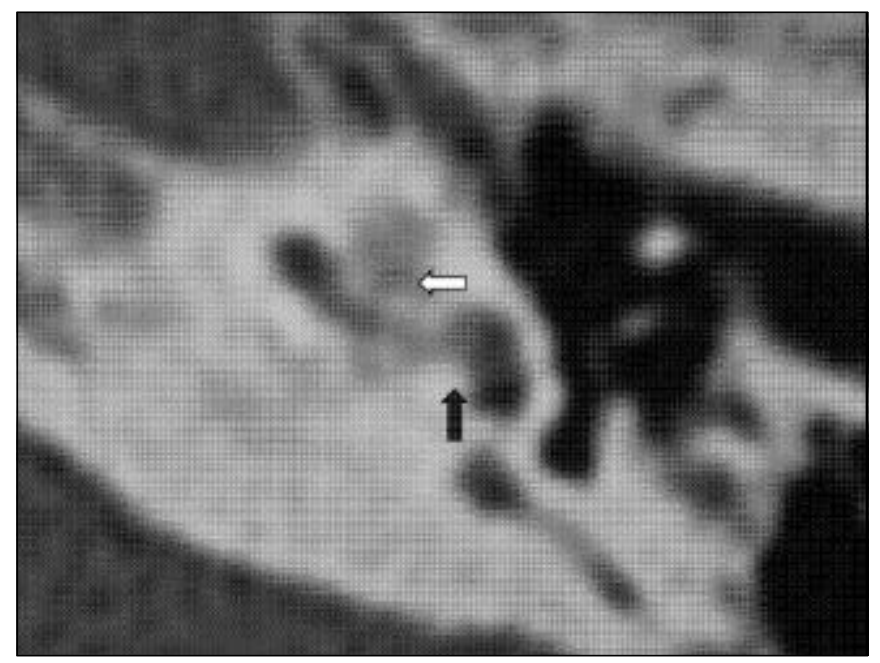

Figura 7.Tomografia computadorizada de orelha esquerda em cortes axiais demonstrando foco hiperdenso no giro basal (seta verde) e hipodenso no giro médio da cóclea (seta)

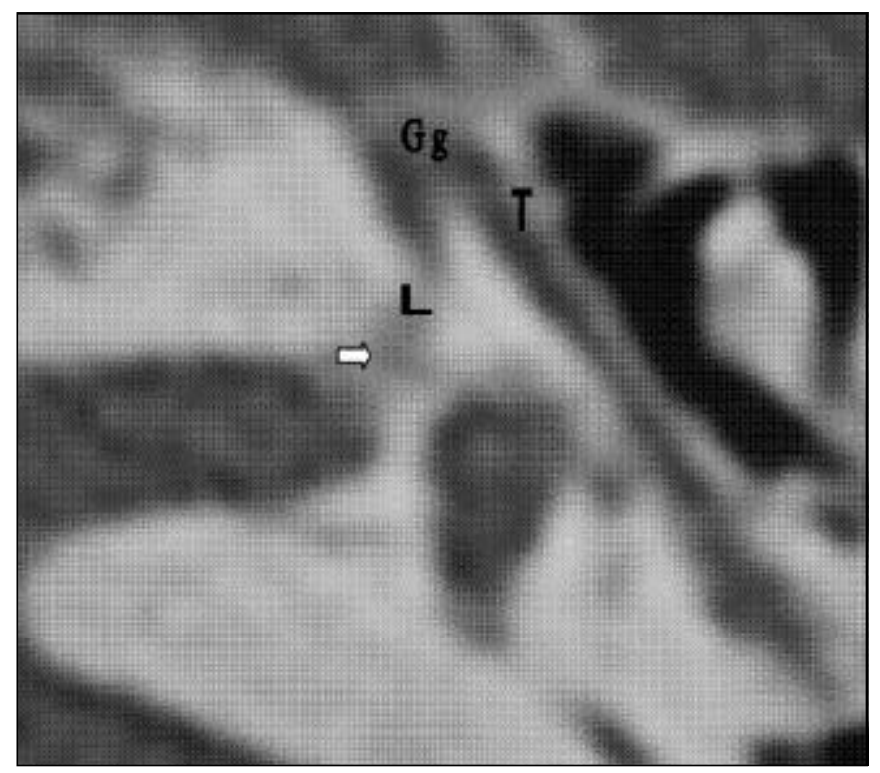

Figura 8. Tomografia computadorizada de osso temporal em corte axial evidenciando foco de hipodensidade e irregularidade no segmento labiríntico do canal do nervo facial e no fundo do meato acústico interno (seta).

T = segmento timpânico do nervo facial; $\mathbf{G g}$ = gânglio geniculado $L=$ segmento labiríntico do nervo facial
Em nossa casuística, um paciente (1,85\%) apresentou foco no segmento labiríntico do nervo facial (FCNFI) na TC (Tabela 4 e Figura 8). A evidência histológica de lesão nessa porção do canal de Falópio foi descrita brevemente por Nager, em casos mais severos de otosclerose ${ }^{17}$. Achamos importante comentar que esse paciente apresentava outros focos otoscleróticos na cápsula ótica e que na sua otoscopia foi evidenciada a presença da mancha de Schwatze, demonstrando, portanto, um grau de atividade maior da doença.

Em nossa casuística não houve diferença estatística entre as duas incidências (coronal e axial) no diagnóstico dos focos retrofenestrais (Tabela 5). Apesar destas evidências, em nossa opinião o corte axial pareceu ser mais fidedigno na avaliação do vestíbulo, do aqueduto coclear e dos giros basal e médio da cóclea.

Em 70 a $90 \%$ dos casos, a otosclerose manifesta-se bilateralmente ${ }^{1,24}$. Em nosso estudo não houve diferença estatisticamente significativa em relação à positividade da TC entre as orelhas, ou seja, os achados tomográficos retrofenestrais foram na maioria dos casos bilaterais. (Tabela 6).

Em quatro pacientes (18,2\%) ou seis orelhas (19,3\%) do nosso grupo controle, evidenciamos a presença da $\mathrm{LPCl}$ (Figura 1 A e B). Tal resultado é similar ao do grupo de pacientes com otosclerose e serve para reforçar a nossa posição de não incluir a LPCI no contexto dos focos otoscleróticos. Foi observada também em um paciente $(4,5 \%)$ a presença de foco de hipodensidade na parede anterior do vestíbulo, em uma orelha $(3,2 \%)$ (Figura 9). Essa evidência tomográfica sugere muito provavelmente a presença de uma lesão otosclerótica silente (histológica) nessa região. Guild, através da análise histológica dos ossos temporais em 1161 pacientes,

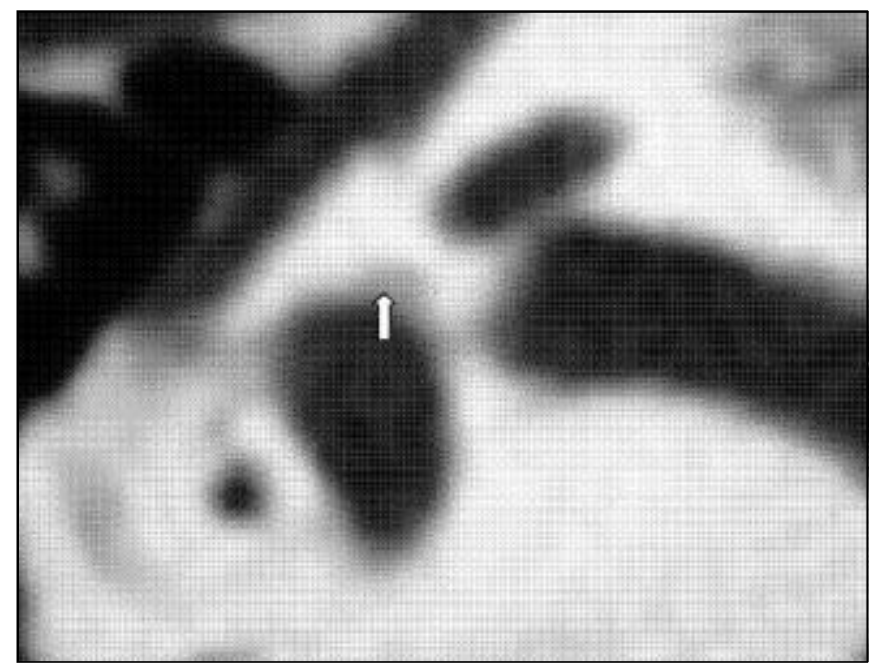

Figura 9. Tomografia Computadorizada em corte axial evidenciando foco de hipodensidade na parede anterior do vestíbulo (seta) em paciente do grupo controle. 
observou que $8,3 \%$ dos caucasianos e $1 \%$ dos negros possuíam evidência histológica de otosclerose, porém a fixação da platina do estribo só ocorria em $12,3 \%$ das orelhas acometidas por essa osteodistrofia ${ }^{29}$. 0 autor enfatizou a importância em distinguir a forma histológica (assintomática) da forma clínica (sintomática) da doença. Na literatura, a prevalência da otosclerose varia de 3 a $12 \%$ na forma histológica e 0,1 a 1\% na forma clínica ${ }^{3,30}$. Nos demais pacientes desse grupo não foram visualizadas anormalidades estruturais da cápsula ótica sugestivas de otosclerose.

\section{CONCLUSÃO}

A tomografia computado rizada mostrou-se eficaz no diagnóstico dos focos otoscleróticos retrofenestrais e a presença dessas lesões deve ser sempre suspeitada e investigada pelo otorrinolaringologista, mesmo em pacientes com indicação cirúrgica.

A evidência tomográfica desses focos juntamente com o envolvimento endosteal da cóclea podem sugerir uma maior atividade da doença. 0 uso de medicações antienzimáticas e antireabsortivas como o fluo reto de sódio e os bifosfonatos deve ser indicado nestes casos.

\section{REFERÊNCIASBIBUOGRÁFICAS}

1. Glasscock ME, Shambaugh GE. Surgery of the Ear. $4^{\text {th }}$ ed, Philadelphia: WB Saunders; 1990. p.371-87.

2. Sorensen MS. Temporal bone dynamics, the hard way: formation, growth, modeling, repair and quantum type bone remodeling in the otic capsule. Acta Otolaryngol Suppl (Stockh) 1994; 512:1-22.

3. Chole RA, McKenna M. Pathophysiology of otosclerosis. Otol Neurotol 2001; 22:249-57.

4. Rovsing H. Otosclerosis fenestral and cochlear. Radiologic Clinics of North America 1974; 12(3):505-15.

5. Swartz JD, Faerber EN, Wolfson RJ, Marlowe FI. Fenestral Otosclerosis: significance of preoperative CT evaluation. Radiology 1984; 151(3):703-7.

6. Valvassori GE: Imaging of the Otosclerosis. Otolaryngol Clin North America 1993; 26(3): 359-71.

7. Maffe MF, Valvassori GE, Deitch RL. Use of CT in cochlear otosclerosis. Radiology 1985a; 156:703-8.

8. Blakley BW, Hilger A, Taylor S, Hilger J. Computed tomography in the diagnosis of cochlear otosclerosis. Otolaryngol Head Neck Surg 1986; 94(4):434-8.

9. Shin YJ, Deguine 0 , Sevely A, Manelfe CL, Fraysse B. Pure Sensorineural hearing loss and otosclerosis. Rev Laryngol Otol Rhinol 2000; 121(2):45-7.
10. Shin YJ, Fraysse B, Deguine O, Cognard C, Charlet JP, Sévely A. Sensorineural hearing loss and O tosclerosis: a clinical and radiologic survey of 437 cases. Acta Otolaryngol 2001a; 121:200-4.

11. Goh JPN, Chan LL, Tan TY.MRI of cochlear otosclerosis. The British Journal of Radiology 2002; 75(894):502-5.

12. Berrettini S, Ravecca F, Volterrani D, Forli F, Boni G, Neri E, Francheschini SS. Single Photon Emission Computed Tomography in Otosclerosis: Diagnostic accuracy and correlation with age, sex, and sensorineural involvement. Otol Neurotol 2002; 23:431-8.

13. D'Archambeau O, Parizel PM, Koekelkoren E, De Heyning V, De Schepper AM. CT diagnosis and differencial diagnosis of otodystrophic lesions of the temporal bone. Eur J Radiol 1990; 11:22-30.

14. Harcourt JP, Lennox P, Phelps MD. CT screening for temporal bone abnormalities in idiopathic bilateral sensorineural hearing loss. J Laryngol Otol 1997; 11:117-21.

15. Valvassori GE, Dobben GD. CT densitometry of the cochlear capsule in otosclerosis. AJNR 1985; 6:661-7.

16. Sonne JE, Zeifer B, Linstrom C. Manifestations of otossífilis as visualized with computed tomography. Otol Neurotol 2002; 23:806-7.

17. Nager GT. Sensorineural deafness and otosclerosis. Ann Otol Rhinol Laryngol 1966; 75: 481-511.

18. Nager GT. Histopathology of otosclerosis. Arch Otolaryng 1969; 89:157-79.

19. Schuknecht HF, Barber W. Histologic Variants in otosclerosis. Laryngoscope 1985; 95:1307-17.

20. Lindsay JR. Histopathology of otosclerosis. Arch Otolaryngol 1973; 97:24-9.

21. Phelps $P$. The basal turn of the cochlea. The British Journal of Radiology 1992; 65:370-4.

22. Ruckenstein MJ, Rafter KO, Montes M, Bigelow DC. Management of far advanced otosclerosis in era of cochlear implantation. Otol Neurotol 2001; 22:471-4.

23. Linthicum FH Jr. Histopathology of otosclerosis. Otolaryngologic clinics of North America 1993; 26(3):335-52.

24. Schucknecht HF: Pathology of the ear. $2^{\text {nd }}$ ed. Philadelphia: Lea $\&$ Febiger; 1993. p.365-79.

25. Veillon F, Riehm S, Emachescu B, Haba D, Roedlich Marie-Noëlle, Greget M, Tongio J. Seminars in Ultrasound, CT, and MRI 2001; 22(3) 271-80.

26. Ghorayeb BY, Linthicum FH Jr. Otosclerosis inner ear syndrome. Ann Otol Rhinol Laryngol 1978; 87:85-90.

27. Takasaki K, Sando Isamu, Hirsch BE. Loss of facial nerve fibers in a case of otosclerosis. Am J Otol 2000; 21:766-8.

28. Swartz JD, Mandell DW, Berman SE, Wolfson RJ, Marlowe FI, Popky GL. Cochlear otosclerosis (otospongiosis): CT analysis with audiometric correlation. Radiology 1985a; 155:147-50.

29. Guild SR. Histologic otosclerosis. Ann Otol Rhinol Laryngol 1944; 53:246-66.

30. Declau F, Van Spaendonck M, Timmermans JP, Michaels L, Liang J, Qiu JP, Van Heyning. Prevalence of otosclerosis in an unselected series of temporal bones. Otol Neurotol 2001; 22:596-602. 